\title{
A lexicographical order induced by Schauder bases
}

\author{
Neşet Özkan Tan (1) \\ Department of Mathematics, Uşak University, 1 Eylül Kampusu, Uşak
}

\begin{abstract}
In this paper, we show that every Banach space with a Schauder basis can be seen as a totally ordered vector space. Indeed, this order can be considered as a lexicographical order since it is a generalization of lexicographical order in $\mathbb{R}^{n}$. We also provide order structural properties of the order by approaching geometrical (cone) sense.
\end{abstract}

Mathematics Subject Classification (2020). 06A05, 49J27, 06A05, 46A45, 46A40

Keywords. total order, cone, Schauder basis

\section{Introduction}

The ordered vector spaces have been studying since at the beginning of the last century and it has efficient applications the other disciplines, see $[1,2,4,5]$. Since the significant properties of the optimization problems in a vector space are frequently based on an order-structure, the optimality concept has been started to approached by the properties of the cone which is a geometric way to understand order structures in the vector spaces. Some of these studies have a wide range of applications such as equilibrium theory and well-posedness problems, see $[7,9-11,16]$.

In this study, we show that we can obtain a totally order by using projections of a Schauder basis of a Banach space that gives us a lexicographical-like order structure. In fact, this cone can be considered as a "generalization" of the lexicographical cone in $\mathbb{R}^{n}$. We also show that the equivalent Schauder basis generates order-isomorphic vector lattices. By associating our findings with some well-known results in Banach space theory, such as every infinite-dimensional Banach space has a subspace that has a Schauder basis [6], we can immediately get the following conclusions: Every separable Banach space has a totally ordered subspace, every infinite-dimensional Banach space has an infinitedimensional quotient space which can be considered as a totally ordered vector lattice. Among the other things mentioned above, we obtain a generalization of the main results of the papers $[9,10]$.

Email address: n.ozkan.tan@gmail.com

Received: 16.12.2019; Accepted: 29.08.2020 


\section{Preliminaries}

Let us recall some of the notions of the ordered vector spaces. In this section, all definitions and aligned properties can be found in [3,4,8,12-14,19]. Throughout of this section, let $E$ be a real vector space and $\theta$ be zero vector in $E$. A subset $\mathcal{K}$ of $E$ is called a cone if: $\mathcal{K}+\mathcal{K} \subset \mathcal{K}, \alpha . \mathcal{K} \subset \mathcal{K}$ for all $\alpha \in \mathbb{R}^{\geq 0}$ and $-\mathcal{K} \cap \mathcal{K}=\{\theta\}$. A subset $\mathcal{W}$ of $E$ is called a wedge if it satisfies all cone axioms except the axiom $-\mathcal{K} \cap \mathcal{K}=\{\theta\}$. The Minkowski sum of $A, B \subseteq E$ is defined by $A+B=\{a+b: a \in A$ and $b \in B\}$ and the scalar multiplication is defined by $\alpha . A=\{\alpha . a: a \in A\}$. It is well known that if $\mathcal{K}$ is a cone in a vector space $E$ then :

$$
a \leq b \text { if and only if } b-a \in \mathcal{K}
$$

is a partial order in $E$. So that, the vector space $E$ with a cone structure $\mathcal{K}$ can be seen as a pair $(E, \mathcal{K})$ which is ordered vector space. Two ordered vector spaces $(E, \mathcal{K})$ and $(M, \mathcal{L})$ is called order isomorphic if there is a linear bijection, $T: E \rightarrow M$ such that $T(\mathcal{K})=\mathcal{L}$. If a pair $(E, \mathcal{K})$ has lattice property (i.e., $\sup \{x, y\}$ or $\inf \{x, y\}$ exists for every pair of $x, y \in E)$ then the pair $(E, \mathcal{K})$ is called an ordered vector lattice or a Riesz space. A sequence $\left\{x_{n}: n \in \mathbb{N}\right\}$ in the ordered vector lattice $E$ is called order convergent to an element $x \in E$ if there exits a monotone decreasing sequence $\left\{q_{n}: n \in \mathbb{N}\right\}$ in $E$ with $\inf \left\{q_{n}\right\}=\theta$ such that $\sup \left\{\left(x_{n}-x\right),\left(x-x_{n}\right)\right\}<q_{n}$ for all $n \in \mathbb{N}$.

If a cone $\mathcal{K}$ has additional property that $-\mathcal{K} \cup \mathcal{K}=E$, then the cone $\mathcal{K}$ is called a totally ordering cone. In this case, the order relation which is induced by the cone structure is called a totally order. Let us introduce some of subspaces of an ordered vector lattice which provide useful information about whole vector lattice. Let $I$ be a subspace of $E$. If $I$ has lattice property then $I$ is called a vector sub-lattice of $E$. If a vector sub-lattice $I$ has solid property (i.e, if a triple of $x, y, y-x \in \mathcal{K}$ with $y \in I$ implies that $x \in I$ ) then $I$ is called an order ideal of $E$. The Minkowski sum of order ideals and intersection of order ideals is an order ideal as well. An order ideal $I$ is called a maximal order ideal if it is the only proper ideal which is contained by itself. Let $(E, \mathcal{K})$ be an ordered vector lattice, $(E, \mathcal{K})$ is called an Archimedean vector lattice if for all $n \in \mathbb{N}$ and for any $x \in E$, $y, y-n x \in \mathcal{K}$ implies that $x=\theta$ or $x \in-\mathcal{K}$. It is well known that the unique totally ordered Achimedean vector lattice is $\mathbb{R}$ with the cone $[0, \infty)$, up to vector lattice isomorphism. The lexicographical order is a non-Archimedean totally order which is defined on $\mathbb{R}^{n \geq 2}$, with the following relation: $\left(x_{1}, x_{2}, \ldots, x_{n}\right)<\left(y_{1}, y_{2}, \ldots, y_{n}\right) \Longleftrightarrow x_{i}<y_{i}$ for the smallest $i$ for which $x_{i} \neq y_{i}$. A sequence $\left(b_{n}\right)$ in a Banach space $X$ is called a Schauder basis of $X$ if for every $x \in X$ there is an unique sequence of scalars $\left(\alpha_{n}\right)$ so that $x=\sum_{n=1}^{\infty} \alpha_{n} . b_{n}$. We should emphasise that for a Schauder basis, there is not only countability, but a specific ordering of base elements. Let $E$ and $L$ be two Banach spaces with Schauder basis $\left(b_{n}\right)$ and $\left(c_{n}\right)$, respectively. Basis $\left(b_{n}\right)$ and $\left(c_{n}\right)$ are called equivalent base if any convergence of $\sum_{n=1}^{\infty} \alpha_{n} . b_{n}$ or $\sum_{n=1}^{\infty} \alpha_{n} \cdot c_{n}$ implies each other.

\section{Totally ordering cones with Schauder basis}

Let $E$ be an infinite dimensional Banach space with Schauder basis $\left(b_{n}\right)$. Each of element $x \in E$ correspond to unique scalar sequence $\left(\alpha_{n}\right)$ where $x=\sum_{n=1}^{\infty} \alpha_{n} . b_{n}$, in the sense of norm convergence. The linear mappings $P_{n}: E \rightarrow E$, defined by

$$
P_{n}(x)=\sum_{k=1}^{n} \alpha_{k} . b_{k} .
$$

Let $b_{n}^{*}: E \rightarrow \mathbb{R}$ denote the functional, where $b_{n}^{*}$ assigns to every vector $x$ in $E$ the coordinate $\alpha_{n}$ of $x$ in the above expansion. Each $b_{n}^{*}$ is a bounded linear functional on $E$. 
Let us define the sequence of sets

$$
\begin{gathered}
B_{1}=\left\{x \in E: b_{1}^{*}(x)>0\right\}, \\
B_{2}=\left\{x \in E: b_{1}^{*}(x)=0 \text { and } b_{2}^{*}(x)>0\right\}, \ldots \\
B_{n}=\left\{x \in E:\left(b_{i}^{*}(x)=0 \text { for all } i<n\right) \text { and } b_{n}^{*}(x)>0\right\}, \ldots
\end{gathered}
$$

If $\mathcal{K}=\bigcup_{n=1}^{\infty} B_{n} \cup\{\theta\}$ then $\mathcal{K}$ is cone in $E$ that produces totally order for the elements of E.

Theorem 3.1. $(E, \mathcal{K})$ is a totally ordered vector lattice.

Proof. We will show that $\mathcal{K}=\bigcup_{n=1}^{\infty} B_{n} \cup\{\theta\}$ is a totally ordered cone. Let us first show that $\mathcal{K}+\mathcal{K} \subset \mathcal{K}$. If at least one of $x, y \in \mathcal{K}$ is zero vector then $x+y \in \mathcal{K}$. If $x \neq \theta$ and $y \neq \theta$ then $b_{i}^{*}(x)>0, b_{j}^{*}(y)>0$ for some $i, j \in \mathbb{N}$, and $b_{n}^{*}(x)=b_{n}^{*}(y)=0$ for all $n<\min \{i, j\}$ Since $b_{k}^{*}$ is a linear functional for all $k \in \mathbb{N}, x+y \in B_{\min \{i, j\}}$, and so $\mathcal{K}+\mathcal{K} \subset \mathcal{K}$. The linearity of $b_{k}^{*}$ 's implies that $\alpha \mathcal{K} \subset \mathcal{K}$ for all $\alpha \geq 0$. Thus $\mathcal{K}$ is a wedge. The linearity of $b_{k}^{*}$ 's also implies that $-\mathcal{K} \cap \mathcal{K}=\{\theta\}$. Now let $\theta \neq x \in E$ then let us define $k:=\min \left\{i \in \mathbb{N}: b_{i}^{*}(x) \neq 0\right\}$. Thus $x \in-B_{k} \cup B_{k} \subset-\mathcal{K} \cup \mathcal{K}$. Therefore, the cone $\mathcal{K}$ is a totally ordering cone in $E$.

The rest of the paper, the notation " $(E, \mathcal{K})$ " means that totally ordered vector lattice with the cone $\mathcal{K}$ which is induced by Schauder basis of the vector space $E$.

Proposition 3.2. Let $E$ and $L$ be two Banach spaces with equivalent basis $\left(b_{n}\right)$ and $\left(c_{n}\right)$, respectively. If $\mathcal{B}$ and $\mathcal{C}$ are totally ordering cones induced by $\left(b_{n}\right)$ and $\left(c_{n}\right)$, respectively, then $(E, \mathcal{B})$ and $(L, \mathcal{C})$ are order isomorphic vector lattices.

Proof. From Closed Graph Theorem, $b_{n}$ and $c_{n}$ are equivalent basis if and only if there is an isomorphism $T: E \rightarrow L$ such that $T\left(b_{n}\right)=c_{n}$ for all $n \in \mathbb{N}$. It is easy to see that for each $x \in E$, we have $b_{n}^{*}(T(x))=c_{n}^{*}(x)$ for all $n \in \mathbb{N}$. Therefore the equality $T(\mathcal{B})=\mathcal{C}$ holds and so, $T$ is an order isomorphism.

The following corollary is immediately obtained from Proposition 3.2 by considering the case $E=L$.

Corollary 3.3. Let $\mathcal{B}$ and $\mathcal{C}$ be totally ordering cones in a Banach space $E$ which are induced by equivalent basis $b_{n}$ and $c_{n}$, respectively. Then $(E, \mathcal{B})$ and $(E, \mathcal{C})$ are order isomorphic vector lattices.

Proposition 3.4. The subset $I_{1}=\left\{x \in E: b_{1}^{*}(x)=0\right\}$ of $E$ is a maximal order ideal in $(E, \mathcal{K})$.

Proof. Firstly, let us show that $I_{1}$ is an order ideal in $(E, \mathcal{K})$. It is not hard to see that $I_{1}$ is a vector sub-lattice of $E$. To show $I_{1}$ has the solid property, let $x, y, y-x \in \mathcal{K}$ with $y \in I_{1}$. Since $b_{1}^{*}(y)=0$ and $b_{1}^{*}(y-x)=b_{1}^{*}(y)-b_{1}^{*}(x)$, then $b_{1}^{*}(x)$ is zero or a negative real number. But the case being negative contradicts with being $x \in K$. Therefore $x \in I_{1}$ and so that $I_{1}$ is an order ideal in $E$.

Now let us show that it is a maximal order ideal. Suppose $L$ is an order ideal in $E$ such that $I_{1} \subsetneq L$. If $x \in L \backslash I_{1}$ then $b_{1}^{*}(x) \neq 0$. We will show that $L=E$. Let us assume that there exists $e \in E \backslash L$, then it is easily to see that $b_{1}^{*}(e) \neq 0$. We can assume that both of $b_{1}^{*}(e)$ and $b_{1}^{*}(x)$ are positive otherwise we can rearrange $-x$ or $-e$ as the positive values. Now, since real numbers are Archimedean there exists $\alpha \in \mathbb{R}$ such that $\alpha b_{1}^{*}(x)>b_{1}^{*}(e)$. The solid property of $L$ implies $e \in L$. Therefore $L=E$ and $I_{1}$ is a maximal order ideal in $(E, \mathcal{K})$.

Indeed, it is not hard to see that $I_{n}=\left\{x \in E: b_{i}^{*}(x)=0\right.$ for all $\left.i \leq n\right\}$ is an order ideal for each $n \geq 1$. Let $I(E)$ be the family of all order ideals in $E$. It is well known that $I(E)$ has a lattice structure if one consider Minkowski sum and intersection as the lattice operations. 
Proposition 3.5. $I(E)$ has countable cardinality.

Proof. We will show that all order ideals of $E$, except itself and $\{\theta\}$, are one of the $I_{n}=\left\{x \in E: b_{i}^{*}(x)=0\right.$ for all $\left.i \leq n\right\}$ for some $n \in \mathbb{N}$. Suppose that a proper order ideal $M \neq I_{n}$ for all $n \geq 1$. Then from maximality of $I_{1}$, it is easy to see that $M \subset I_{1}$. Otherwise, by following second part of proof of Proposition 3.4, $M$ must contain all elements of $E$. Indeed, $M$ should be also a subset of $I_{2}$. If it is between $I_{1}$ and $I_{2}$ then again by following second part of proof of Proposition 3.4, $M$ should be equal $I_{1}$. Now, one can get the desired result by induction over $n \geq 1$. Therefore all order ideals of $E$ must be equal one of $\left\{I_{n}\right\},\{\theta\}$ or $E$.

Corollary 3.6. The lattice $I(E)$ is totally ordered.

It is well known that if $I$ is an order ideal in a vector lattice $E$, then the quotient vector space $E / I$ is a vector lattice with the following order : $\phi(x)>0$ if $x+y>\theta$ for all $y \in I$, where $\phi$ is the canonical map from $E$ to $E / I$. If $I$ is a maximal order ideal in a vector lattice $E$ then the quotient vector lattice $E / I$ is order isomorphic to the real numbers, see [17]. So the following corollary is obtained immediately from the proof of Proposition 3.5, since $I_{1}$ is the unique maximal ideal of $E$ we have the following corollary.

Corollary 3.7. E/I $/ I_{1}$ is lattice isomorphic to $\mathbb{R}$.

The cone $\mathcal{K}$ is not Archimedean (A totally ordered cone is closed if and only if it has at most 1 dimension, see [4]), nevertheless, we have following relationship between order convergence and base projections.

Lemma 3.8. If a sequence $\left\{x_{n}\right\}$ of $E$ is order convergent to $x \in E$, then the real sequence $\left\{b_{k}^{*}\left(x_{n}-x\right)\right\}$ converges to zero for each $k \in \mathbb{N}$.

Proof. First of all, let us show that if $q_{n} \downarrow \theta$ in $E$, then $b_{k}^{*}\left(q_{n}\right) \downarrow 0$ for each $k \in \mathbb{N}$. Let us assume that $q_{n} \downarrow \theta$ in $E$ but $r:=\inf _{n \in \mathbb{N}} b_{k_{0}}^{*}\left(q_{n}\right) \neq 0$ for a $k_{0} \in \mathbb{N}$. We can assure that this infimum exits because of that the sequence $b_{k_{0}}^{*}\left(q_{n}\right)$ is bounded below from zero. Let $y$ be chosen such that $0<b_{k_{0}}^{*}(y)<r$ and $b_{k}^{*}(y)=0$ for all $k<k_{0}$. Then obviously $y \neq \theta$ and $q_{n}>y$ for all $n \in \mathbb{N}$ which contradicts with being $q_{n} \downarrow \theta$.

Now, let $x_{n}$ be order convergent to $x \in E$. Then there exits a sequence $q_{n} \downarrow \theta$ such that $\left|x_{n}-x\right|<q_{n}$ for each $k \in \mathbb{N}$. From the inequality $b_{k}^{*}\left(\left|x_{n}-x\right|\right)<b_{k}^{*}\left(q_{n}\right)$ and with the previous observation, we obtain that the sequence $b_{k}^{*}\left(\left|x_{n}-x\right|\right)$ converges to zero for each $k \in \mathbb{N}$. By using linearity of $b_{k}^{*}$, we can easily get the desired result.

Example 3.9. The norm convergence does not imply the order convergence and vice versa. Let us consider the Banach space $c_{0}$ with sup norm. Now consider sequence of $x_{n}=\left(\frac{1}{n}, 0,0, \ldots\right)$ for $n \in \mathbb{N}$. It is easy to see that the sequence $\left\{x_{n}: n \in \mathbb{N}\right\}$ converges to zero with sup norm. But it does not order converge to zero. To see this, it is enough to observe that $\inf _{n \in \mathbb{N}}\left\{x_{n}\right\}>(0,1,0,0, \ldots)>\theta$.

In order to see that order convergence does not imply norm convergence, let us consider the Schauder basis $\left(e_{n}\right)_{n=1}^{\infty}$ of $c_{0}$ which is not a Cauchy sequence with respect to sup norm, but it is order convergent to zero vector. It is clear that zero vector is a lower bound for the sequence $\left\{e_{n}\right\}$ and let us assume that $e \in E$ is another lower bound for $\left\{e_{n}\right\}$ such that $e>\theta$. Since $e>\theta$ then there exits an integer $n_{0}$ such that $n_{0}$ th term of the sequence $e$ is a positive real number. But in this case we obtain $e_{n_{0}+1}<e$ and this contradict with property of $e$ that being lower bound of $\left\{e_{n}\right\}$. Therefore $\theta$ is greatest lower bound of $\left\{e_{n}\right\}$, so that it is order convergent to zero vector.

It is well known that Hamel base of the finite dimensional Banach spaces can be seen as a Schauder basis and they are all equivalent to Hamel base of $\mathbb{R}^{n}$. Since there is only 
one totally ordering cone in $\mathbb{R}^{n}$ and by Proposition 3.2 , we can re-state the following well-known corollary.

Corollary 3.10. Every finite dimensional totally ordered vector lattice is order isomorphic to $\left(\mathbb{R}^{n},<_{\text {lex }}\right)$.

Indeed, it is well known that in a Hilbert space, all orthonormal basis are equivalent. Since every orthogonal base in a separable Hilbert space can be seen as a Schauder basis, Proposition 3.2 gives us the following corollary which is the main result of [10].

Corollary 3.11. Every separable Hilbert space has totally ordering cone.

\section{References}

[1] C.D. Aliprantis, C. Bernard and R. Tourky Economic equilibrium: Optimality and price decentralization, Positivity, 6 (3), 205-241, 2002.

[2] C.D. Aliprantis and O. Burkinshaw, Locally solid Riesz spaces with applications to economics, American Mathematical Society, Providence, RI, 2003.

[3] C.D. Aliprantis and O. Burkinshaw, Positive Operators, Springer, Dordrecht, 2006.

[4] C.D. Aliprantis and R. Tourky, Cones and duality, 84, American Mathematical Society, Providence, RI, 2007.

[5] C.D. Aliprantis, R. Tourky and C.Y. Nicholas, Cone conditions in general equilibrium theory, J. Econom. Theory 1, 96-121, 2000.

[6] C. Bessaga and A. Pelczynski, On bases and unconditional convergence of series in Banach spaces, Studia Math. 17, 151-164, 1958.

[7] M. Ehrgott, Multicriteria optimization, Springer Science and Business Media, 2005.

[8] E. Jonge and A.C.M Rooij, Introduction to Riesz spaces, Mathematisch Centrum, 1977.

[9] M. Kucuk, M. Soyertem and Y. Kucuk, On constructing total orders and solving vector optimization problems with total orders, J. Global Optim. 50, (2), 235-247, 2011.

[10] M. Kucuk, M. Soyertem and Y. Kucuk, The generalization of total ordering cones and vectorization to separable Hilbert spaces, J. Math. Anal. Appl. 389, (2), 1344-1351, 2012.

[11] M. Kucuk, M. Soyertem, Y. Kucuk and I. Atasever, Vectorization of set-valued maps with respect to total ordering cones and its applications to set-valued optimization problems, J. Math. Anal. Appl. 385 (1), 285-292, 2012.

[12] J. Lindenstrauss and L. Tzafriri, Classical Banach Spaces I-II Function Spaces, Springer, 1996.

[13] W.A. Luxemburg and A.C. Zaanen, Riesz Spaces I, North-Holland, Amsterdam, 1971.

[14] N.P. Meyer, Banach Lattices, Springer, Berlin, 1991.

[15] A. Pelczynski and I. Singer, On non-equivalent basis and conditional basis in Banach spaces, Studia Math. 1 (25), 5-25, 1964.

[16] A.L. Quoc and D.T. Quoc, Tykhonov well-posedness for lexicographic equilibrium problems, Optimization, 65 (11), 1929-1948, 2016.

[17] H.H. Schaefer, Banach lattices and positive operators, Springer-Verlag, 1974.

[18] J. Schauder, Zur Theoriestetiger Abbildungenin Funktionalraumen, Math. Z. 26, 4765, 1927.

[19] A.C. Zaanen, Introduction to Operator Theory in Riesz Spaces, Springer-Verlag, 1997. 